\title{
Iliac Artery Lesion as a Severe Complication of Hysteroscopic Myomectomy
}

\author{
Daniela Angerame Yela, MD, PhD, Patrícia Kajikawa, MD, Cassia Raquel Juliato, MD, PhD, \\ Cristina Laguma Benetti-Pinto, MD, PhD \\ Department of Gynecology and Obstetrics, School of Medical Sciences, University of Campinas, Campinas, \\ São Paulo, Brazil (all authors).
}

\begin{abstract}
Hysteroscopic myomectomy is the surgical procedure of choice for the treatment of submucous or intramural myomas that protrude into the uterine cavity in patients with abnormal bleeding and/or infertility. It is a minimally invasive procedure associated with a low complication rate. Complications of hysteroscopy include uterine perforation, intraoperative and postoperative uterine bleeding, water intoxication, gas embolism, and injuries of the bladder, ureters, and major blood vessels, in addition to late complications, such as infections and synechiae. This case report describes iliac artery injury during hysteroscopic myomectomy. A review of the literature shows that this complication has not been recorded.
\end{abstract}

Key Words: Hysteroscopy, Myomectomy, Complications, Iliac artery injury

Citation Yela DA, Kajikawa P, Juliato CR, Benetti-Pinto CL. Iliac artery lesion as a severe complication of hysteroscopic myomectomy. CRSLS e2014.00333. DOI 10.4293/CRSLS.2014.00333.

Copyright (C) 2014 SLS This is an open-access article distributed under the terms of the Creative Commons Attribution-Noncommercial-ShareAlike 3.0 Unported license, which permits unrestricted noncommercial use, distribution, and reproduction in any medium, provided the original author and source are credited.

Address correspondence to: Daniela Angerame Yela, Departamento de Tocoginecologia, Faculdade de Ciências Médicas, Universidade Estadual de Campinas UNICAMP, Rua Alexander Fleming n¹01, CEP:13083-970, Campinas, SP, Brazil; Telephone/fax: 55-19-3521-9306, E-mail: yela@unicamp.br.

\section{INTRODUCTION}

Hysteroscopy is a procedure that is routinely performed in gynecologic practice because of its diverse indications, such as premenopausal or postmenopausal abnormal uterine bleeding, infertility, recurrent abortions, pain, and alterations on imaging examinations in asymptomatic women. It is a minimally invasive procedure associated with a low complication rate. If used only for diagnosis, the process is limited to the visualization with or without sampling of myometrial or cervical canal lesions. Complication rates in diagnostic hysteroscopy are very low, at about $0.13 \% .{ }^{1}$ Hysteroscopy is also used for intrauterine surgery as lysis of adhesions, metroplasty, myomectomy, polypectomy, sterilization, and endometrial ablation. Surgical hysteroscopy carries a higher risk for complications, ranging from $0.95 \%$ to $3.5 \%$, as recorded in the literature. ${ }^{1-3}$

Hysteroscopic myomectomy is the surgical procedure of choice for the treatment of submucous or intramural myomas that protrude into the uterine cavity in patients with abnormal bleeding and/or infertility. ${ }^{4}$ First performed by Neuwirth in 1976, hysteroscopic myomectomy has be- come essential in the treatment of submucous myomas, especially among young women who desire to maintain fertility. ${ }^{5}$ Although considered a less invasive surgical procedure, along with metroplasty, hysteroscopic myomectomy has the highest risk for complications, with a prevalence rate of up to $10 \%{ }^{6}$

Complications of hysteroscopy may be related to cervical dilatation, distention medium with gas embolism, or to water intoxication. Mechanical complications are also possible, such as uterine perforation and injuries caused by instruments (e.g., lesions of the bladder, ureters, and major blood vessels). Also reported have been uterine bleeding and late complications, such as infections and synechiae.

This case report describes iliac artery injury during hysteroscopic myomectomy. In a review of the literature, we determined that this complication has not previously been recorded.

\section{CASE REPORT}

The patient was a Caucasian, 18-year-old nulliparous woman, receiving outpatient follow-up care for heavy 
menstrual bleeding (cycles lasting 7-10 d with clots) for 1 year. Ultrasound showed a submucous myoma measuring $1.1 \mathrm{~cm}$. Diagnostic hysteroscopy revealed a grade 2 submucous fundal myoma, measuring $3 \mathrm{~cm}$. The patient underwent hysteroscopic myomectomy using a monopolar system, with a partial resection of $70 \%$ of the myoma. The procedure was discontinued after 42 minutes because of risk for water intoxication with the use of $6 \mathrm{~L}$ of glycine $1.5 \%$. In the immediate postoperative period, the patient progressed with tachycardia, tachypnea, pale mucosa, and painful abdominal distension. Because the patient was in shock, an exploratory laparotomy of the abdominal cavity was indicated, which showed a large amount of free blood, approximately $2.5 \mathrm{~L}$, with a $0.5-\mathrm{cm}$ perforating lesion in the posterior uterine wall with profuse active bleeding originating from a right retroperitoneal lesion. A lesion of the right internal iliac artery measuring about 0.5 $\mathrm{cm}$ was identified and repaired by the vascular surgeon. Twelve hours postoperatively, the patient presented with hemodynamic instability and underwent reexploration of the abdominal cavity. Retroperitoneal bleeding was diffuse. Bleeding was controlled using 5 large pads for packing, and peritoneostomy was performed to close the abdominal cavity temporarily. After the correction of a number of coagulation disorders (transfusion of $26 \mathrm{U}$ of red blood cell concentrates, $25 \mathrm{U}$ of fresh frozen plasma, $8 \mathrm{U}$ of platelet concentrates, factor VIIA, and fibrinogen), the 5 pads were removed, and the abdominal cavity was closed. The patient's clinical course progressed with sepsis and acute pancreatitis. The patient was discharged after 34 days of hospitalization. She returned to the outpatient clinic after 40 days, reporting normal menstrual flow lasting 5 days and mild dysmenorrhea. Ultrasound revealed a 101-mm uterine volume with no delimitation of myomatous nodules. After 2 years of follow-up, the patient remains asymptomatic, with a regular menstrual cycle of 3 to 4 days, and takes combined oral contraceptives. Transvaginal ultrasound revealed an $83.5-\mathrm{cm}^{3}$ uterus and an anechoic cystic image measuring $6 \times 6 \mathrm{~mm}$, which may correspond to a myometrial cyst, without myomas.

\section{DISCUSSION}

Hysteroscopic myomectomy may be considered the least invasive surgical procedure for the treatment of submucous myoma. However, this procedure has the highest risk for complications among hysteroscopies. The complication rate in hysteroscopic myomectomy ranges from $0.3 \%$ to $5.3 \% \%^{7}$ and seems to be dependent on the surgeon and technique used.
Complications due to hysteroscopic myomectomy may occur at several time points in the procedure: during anesthesia, cervical dilatation, distension of the uterine cavity, resectoscope insertion, and myoma resection with monopolar or bipolar technology. Late complications, such as infections, synechiae, and fistulas, may occur after surgical performance. Munro ${ }^{8}$ summarized the most common complications. Hysteroscopic complications can result from patient positioning (neurologic lesions), anesthesia, access to hysteroscopy (cervical trauma and perforation uterine), and distention medium (gas embolism, fluid overload, and electrolyte disturbances). Other complications include uterine bleeding, injuries caused by instruments and electrosurgical devices (bladder lesions, ureter lesions, and major blood vessel lesions), and infection. Late complications include synechiae and pregnancy complications such as uterine rupture, placenta accreta or increta, and others. ${ }^{8}$

The most prevalent and severe clinical complication during hysteroscopic myomectomy is excess intravascular absorption of the fluid used for uterine cavity distension and irrigation. Severe fluid overload may cause pulmonary edema, hyponatremia, heart failure, cerebral edema, and death. ${ }^{9}$ Fluid absorption occurs through open veins in the myoma and possibly transperitoneally by retrograde fluid absorption through the uterine tubes. The risk factors for intoxication during hysteroscopic myomectomy have not been fully elucidated. The main risk factor seems to be intramural extension of the myoma. ${ }^{9}$ Other factors reported may include prolonged surgical time, characteristics of the distending media, and pressure produced by the irrigation system.

The most frequent surgical complication is uterine perforation, which may occur during cervical dilatation, hysteroscopic insertion, and myometrial tissue resection. In general, the risk for perforation increases in case of myomas, particularly those close to the uterine cornua, where the myometrium may be $<4 \mathrm{~mm}$ thick. The risk is also associated with the intramural component of the myoma. ${ }^{10} \mathrm{We}$ currently use the classification system developed by Wamsteker et $\mathrm{al}^{11}$ in 1993 and adopted by the European Society for Gynecological Endoscopy, which classifies submucous myomas according to the degree of myometrial penetration (G0, G1, or G2). A G0 myoma is located entirely within the endometrial cavity and appears to be attached to the cavity wall only by a fine pedicle; a G1 myoma protrudes $>50 \%$ into the uterine cavity; and a G2 myoma penetrates $>50 \%$ into the myometrium. Therefore, the higher the myoma score, the higher the risk for uterine perforation. 
Another classification for submucous myomas is termed STEP-w. It categorizes myomas into 3 groups: I (score 0-4), II (score 5-6), and III (score 7-9). Scoring is obtained by assessment of myoma size, myoma topography, extension of the base of the myoma, penetration, and whether the myoma is located on the lateral wall of the uterus. The complexity level of hysteroscopic myomectomy varies according to group (group I, low-complexity hysteroscopic myomectomy; group II, high-complexity hysteroscopic myomectomy [consider gonadotropin-releasing hormone use, consider 2-step hysteroscopic myomectomy]; group III, consider alternatives to the hysteroscopic technique). ${ }^{12}$

A study of 205 hysteroscopic myomectomies showed that complications such as fever, perforation, pain, bleeding, and water intoxication occurred in 15 cases (7.3\%). According to the STEP-w classification, 7 complications occurred in myomas scoring $\leq 4$ and 8 in those scoring $>4 .{ }^{13}$

Uterine perforation, the most frequent hysteroscopic complication, usually results only in the inability to maintain uterine distension. Therefore, the procedure is prematurely discontinued. However, this perforation may be related to severe damage to pelvic viscera and blood vessels. When perforation occurs and there is evidence of bleeding or the possibility of visceral lesion, a laparoscopy or laparotomy is indicated. In our case, there was a uterine perforation that was not seen in intraoperatively. This probably occurred because of heavy bleeding and difficulty in intracavitary visualization, which delayed the diagnosis of iliac artery injury.

In 2000, Stotz et $a^{14}$ described the case of a patient undergoing hysteroscopic myomectomy complicated by right iliac vein injury. The authors emphasized that abdominal bleeding was masked by hemodilution, delaying and making the diagnosis difficult. During laparotomy, 2 areas of necrosis in the posterior uterine wall were observed. It was questioned whether the iliac lesion had occurred by direct resectoscopic trauma or an indirect thermal lesion. A thermal lesion, usually also associated with uterine perforation, can be due to either monopolar or bipolar technologies and may also damage the bladder, bowel, and major blood vessels. In our case, similar to that reported by Stotz et al describing iliac vein injury, the mechanism of lesion (whether mechanical or thermal) remains unknown.

Bahar et $\mathrm{al}^{15}$ evaluated 1,842 hysteroscopic procedures. Bipolar technology was used in 1,318 of these surgeries. No statistically significant difference was demonstrated in the complication rate when monopolar and bipolar technologies were compared. Laparotomy was performed in 5 patients $(0.3 \%), 2$ of whom required hysterectomies. Both hysterectomies were performed in patients who had previously undergone hysteroscopic myomectomies. Furthermore, the authors noted that uterine perforation involved a major blood vessel injury in 1 patient, requiring assistance from the vascular surgery team. However, the authors failed to specify the vessel damaged or the technique used.

Although several studies have shown no differences in complications of myomectomy related to the type of energy used (monopolar or bipolar), it is known that monopolar current dissipates more than bipolar current, which could have contributed to the injury. ${ }^{16}$ Some studies have shown that the use of cold loops to remove the intramural portion of a myoma could decrease complications arising from the electric current. ${ }^{17}$

To prevent complications in myomectomy, the literature suggests that a good presurgical evaluation is required. The most common modality used to evaluate a fibroid is transvaginal ultrasound. It is important to assess the size of the fibroid besides the myometrial-free margin (for safe hysteroscopic resection, the margin must be $\geq 1 \mathrm{~cm}$ ). Sonohysterography demonstrates better accuracy for the diagnosis of myoma. In patients with large uteri with multiple fibroids or if ultrasound is technically difficult (as in obese patients), magnetic resonance imaging may be a good option. ${ }^{17}$

The use of gonadotropin-releasing hormone agonists before myomectomy can reduce the endometrial thickness and the size and vascularization of fibroids. Some studies recommend its use in myomas larger than $2 \mathrm{~cm}$, but other studies suggest that there are no advantages before myomectomy. ${ }^{17}$ In our case, the use of a gonadotropin-release hormone agonist may have prevented this complication by reducing bleeding and improving visualization.

Thus, a good presurgical evaluation and a skilled and experienced surgeon using suitable material can minimize complications in myomectomy.

\section{CONCLUSIONS}

Surgical hysteroscopy is considered a routine minimally invasive surgical procedure. However, it is not devoid of complications. The most common complications are hemorrhage, uterine perforation, and water intoxication. Major blood vessel injury is a severe complication of surgical hysteroscopy, with few cases described in the literature. 
No previous case report of iliac artery lesion was found. Despite the high morbidity and mortality risk related to the procedure, adequate multidisciplinary medical care may produce a favorable outcome.

\section{References:}

1. Jansen FW, Vredevoogd CB, van Ulzen K, Hermans J, Trimbos JB, Trimbos-Kemper TC. Complications of hysteroscopy: a prospective, multicenter study. Obstet Gynecol. 2000;96(2):266270.

2. Shveiky D, Rojansky N, Revel A, Benshushan A, Laufer N, Shushan A. Complications of hysteroscopic surgery: "beyond the learning curve". J Minim Invasive Gynecol. 2007;14(2): $218-222$.

3. Agostini A, Bretelle F, Cravello L, Ronda I, Roger V, Blanc B. Complications of operative hysteroscopy [article in French]. Press Med. 2003;32(18):826-829.

4. Agdi M, Tulandi T. Endoscopic management of uterine fibroids. Best Pract Res Clin Obstet Gynaecol. 2008;22(4):707716.

5. Neuwirth RS, Min HK. Excision of submucous fibroids with hysteroscopic control. Am J Obstet Gynecol. 1976;26:95-99.

6. Propst AM, Liberman RF, Harlow BL, et al. Complications of hysteroscopic surgery: predicting patients at risk. Obstet Gynecol. 2000;96(4):517-520.

7. Batra N, Khunda A, O'Donovan PJ. Hysteroscopic myomectomy. Obstet Gynecol Clin North Am. 2004;31:669-685.

8. Malcolm G. Munro. Complications of hysteroscopic and uterine resectoscopic surgery. Obstet Gynecol Clin North Am. 2010;37(3):399-425.
9. Emanuel MH, Wamsteker K. Uterine leiomymas. In: Brosens I, Wamsteker K, eds. Diagnostic Imaging and Endoscopy in Gynecology. London: WB Saunders; 1997:185-198.

10. Murakami T, Tamura M, Ozawa Y, Suzuki H, Terada Y, Okamura K. Safe techniques in surgery for hysteroscopic myomectomy. J Obstet Gynaecol Res. 2005;31(3):216-223.

11. Wamsteker K, Emanuel MH, de Kruif JH. Transcervical hysteroscopic resection of submucous fibroids for abnormal uterine bleeding: results regarding the degree of intramural extension. Obstet Gynecol. 1993;82(5):736-740.

12. Lasmar RB, Barrozo PRM, Dias R, Oiveira MAP. Submucous fibroids: a new presurgical classification (STEP-w). J Minim Invasive Gynecol. 2005;12:308-311.

13. Lasmar RB, Lasmar BP, Celeste RK, da Rosa DB, Depes Dde B, Lopes RG. A new system to classify submucous myomas: a Brazilian multicenter study. J Minim Invasive Gynecol. 2012; 19(5):575-580.

14. Stotz M, Lampart A, Köchli OR, Schneider M. Intraabdominal bleeding masked by hemodilution after hysteroscopy. Anesthesiology. 2000;93(2):569-570.

15. Bahar R, Shimonovitz M, Benshushan A, Shushan A. Casecontrol study of complications associated with bipolar and monopolar hysteroscopic operations. J Minim Invasive Gynecol. 2013;20(3):376-380.

16. Capmasa P, Levaillanta J M, Fernandez H. Surgical techniques and outcome in the management of submucous fibroids. Curr Opin Obstet Gynecol. 2013;25(4):332-338.

17. Sardo ADS, Mazzon I, Bramante S, et al. Hysteroscopic myomectomy: a comprehensive review of surgical techniques. Hum Reprod Update. 2008;14(2):101-119. 